\title{
COMUNIDADES DE APRENDIZAJE: PROPUESTA DE DESARROLLO Y SOSTENIBILIDAD DESDE LA EDUCACIÓN SOCIAL EN INSTITUCIONES EDUCATIVAS.
}

\author{
Learning communities: proposal for development and sustainability from the \\ social education in educational institutions.
}

\author{
Ana Rocio Jiménez González \\ María del Rocio Rodríguez Casado
}

Fecha recepción: 15/07/2016

Fecha aceptación: 17/11/2016

RESUMEN: Con el presente artículo ofrecemos una visión desde la Educación Social para con los centros escolares que están inmersos en el modelo educativo de las Comunidades de Aprendizaje, ya que a pesar de tratarse de una práctica que aparece hace más de 35 años en España (Morlà 2015), aún es bastante desconocida dentro del ámbito escolar. Esto hace que podamos encontrar muchas lagunas en su implantación, y sobre todo, en el mantenimiento del mismo con el paso de los años. Es por ello que, a continuación, presentamos una propuesta socioeducativa para las Comunidades de Aprendizaje desde la Educación Social como modelo de desarrollo y sostenibilidad, donde prima la intervención con toda la comunidad educativa en su conjunto de forma interrelacional. En este sentido, entendemos que la educación del alumnado es responsabilidad de toda su comunidad, y aunque un proyecto sea excelente, siempre ha de tener previsto un procedimiento o protocolo que lo pueda sustentar cuando el proyecto vaya perdiendo entusiasmo. Si bien, el propósito de esta publicación va más allá, pues se intenta hacer una reflexión sobre el papel que juega el/la educador/a social en el desarrollo y sostenibilidad de las Comunidades de Aprendizaje en los centros escolares.

PALABRAS CLAVE: Comunidad de Aprendizaje, centros educativos, sostenibilidad, Educación Social.

ABSTRACT: In this article we offer a view from the Social Education with schools that are involved in the educational model Learning Communities because despite being a practice that occurs more than 35 years ago in Spain (Morlà, 2015) it is still quite unknown in the schools. This allows us to find many gaps in its implementation, and especially in its maintenance over the years. That is why, then present a proposal for Learning Communities from Social Education as a model of development and sustainability, where raw intervention with the entire educational community as a whole so inter-relational. In this sense, we understand that the education of students is the responsibility of the entire community, and although a project is excellent, always must have provided a method or protocol that can support when the project is losing enthusiasm. While the purpose of this publication goes further, as it tries to reflect the active role played by social educators in the development and sustainability of learning communities in schools.

KEYWORDS: Learning Community, schools, sustainability, Social Education. 


\section{Introducción}

$\mathrm{Si}$ echamos la vista atrás, independientemente de las diversas y continuas modificaciones sufridas en nuestra sociedad desde diferentes perspectivas (sociales, económicas, políticas, tecnológicas, religiosas, culturales, ideológicas, de valores...), podemos observar como nuestro sistema educativo apenas ha cambiado a lo largo de la historia. Así, teniendo en cuenta todos los avances que las sociedades han ido experimentando en las últimas décadas, se hace necesaria una adaptación y renovación del sistema escolar a los nuevos desafíos que se generan en nuestra sociedad. La escuela como institución educativa necesita una transformación con miras hacia lo social, ya que todo cambio en la sociedad influye y, sobre todo, afecta en el ámbito escolar y por ende a toda la comunidad educativa, entendida como toda persona que participa para con la escuela (alumnado, profesorado, miembros directivos, familias, persona de mantenimiento y apoyo, voluntariado, otros agentes educativos y profesionales internos y/o externos, el vecindario, etc.).

Estamos hablando, en este sentido, de compartir el espacio educativo con la vida cotidiana del alumnado, su familia, su entorno, etc... Desde este enfoque de apertura, flexibilidad y comprensión de todos los cambios acaecidos en nuestra sociedad profundizamos en el modelo educativo de las Comunidades de Aprendizaje desde la Educación Social que evoluciona la idea misma de educación, ajustándose a las nuevas necesidades fraguadas actualmente. De esta forma, esta nueva perspectiva de las Comunidades de Aprendizaje facilitaría espacios de verdadera convivencia, extendiendo a la escuela la posibilidad de diálogo, las relaciones de igualdad y el aprendizaje mutuo. Por lo que basándonos en Díez-Palomar y Flecha (2010), podemos decir que se trataría de una respuesta eficaz y equitativa a dichos cambios que afectan en el interior de las instituciones educativas.

Concretamente, este tema resulta interesante desde la perspectiva de la Educación Social, ya que entre sus muchos ámbitos de intervención, el sistema escolar es uno de los menos reconocidos en el marco de su profesionalización. En Andalucía, es a partir de la publicación de las Instrucciones de la Dirección General de Participación e Innovación Educativa de la Junta de Andalucía $(2010,2)$ por las que se regula la intervención del educador y educadora social en el ámbito educativo. Pues, según su primera instrucción "se incorporan al sistema educativo como profesionales especializados en la intervención personal, social y familiar, constituyendo un recurso y un apoyo necesario para mejorar las acciones formativas en los Centros y en las Comunidades Educativas".

Por lo que desde esta contribución defendemos la necesidad de la participación de los/as educadores/as sociales dentro de la realidad escolar, incorporándose dentro del modelo de las Comunidades de Aprendizaje como una oportunidad más para reconocer y valorizar su profesión, en la que se demuestra la importante labor de este perfil como agente de cambio social,

Por otro lado, aunque según ley el profesional en educación social se adscriba en los Equipos de Orientación Educativa caracterizados por su interdisciplinariedad y especialización de sus actuaciones, por su apoyo a la actividad docente, por su perspectiva de zona y contribución a la dinamización e innovación educativa (Ley 17/2007, de 10 de diciembre de Educación de Andalucía), consideramos que resulta muy enriquecedor elaborar una propuesta socioeducativa de la mano del equipo docente, el cual, en muchas ocasiones, desconoce la figura del/la educador/a social y presentan una actitud de rechazo hacia su labor dentro del centro escolar. Por lo que a partir de este modelo socioeducativo, se plantea una intervención interdisciplinar para trabajar conjuntamente sobre las necesidades en las 
escuelas, especialmente, sobre el desarrollo y el sostenimiento de las Comunidades de Aprendizaje en instituciones educativas.

En definitiva, continuar trabajando desde las Comunidades de Aprendizaje en una institución educativa no es una tarea fácil. A pesar de las dificultades, en la actualidad, podemos contar con Comunidades de Aprendizaje en Andalucía, Aragón, Castilla La Mancha, Castilla León, Cataluña, Extremadura, Murcia, Galicia, Madrid, Navarra, La rioja, Comunidad Valenciana, Ceuta y Melilla (Gómez et al., 2010; Morlà, 2015). Sin embargo, nuestro planteamiento gira en torno a la reflexión que nos hacemos como profesionales de la educación social sobre las siguientes cuestiones: ¿qué se entiende por Comunidad de Aprendizaje? ¿Cuándo se implementó la Comunidad de Aprendizaje en los centros escolares de dichas regiones autonómicas? ¿Qué instituciones educativas son las que apuestan por la implantación de las Comunidades de Aprendizaje? ¿Qué perfiles profesionales trabajan por y para la Comunidad de Aprendizaje? ¿Qué estrategias se llevan a la práctica para el desarrollo y la sostenibilidad de este modelo socioeducativo? ¿Cuál es el rol que ocupa la comunidad educativa con respecto a la Comunidad de Aprendizaje?

\section{Las comunidades de aprendizajes en instituciones educativas.}

Con objeto de definir la concepción de Comunidad de Aprendizaje, podemos decir que se entiende por "escuela inclusiva fundamentada en el trabajo en red, que presenta un proyecto integrador e integrado abierto a la participación de todos los agentes vinculados con la escuela" (Ferrer, 2005, 62). Asimismo, traemos a colación las palabras de Valls $(2000,8)$ que la define como

[...] un proyecto de transformación social y cultural de un centro educativo y de su entorno para conseguir una sociedad de la información para todas las personas, basada en el aprendizaje dialógico, mediante una educación participativa de la comunidad, que se concreta en todos sus espacios incluida el aula (Valls, 2000, 8).

Para Morlà (2015, 139), las Comunidades de Aprendizaje surgió como movimiento educativo y proyecto "basado en un conjunto de Actuaciones Educativas de Éxito (AEE) fundamentadas en evidencias científicas para dar respuesta a los nuevos retos sociales, ofreciendo una respuesta educativa igualitaria y trabajando por la cohesión social". Aunque expone que no siempre ha sido un modelo teórico defendido, se evidencia tras su práctica que la participación de toda la comunidad educativa dentro de la escuela mejora el aprendizaje del alumnado (citado en Flecha y Soler, 2013).

A partir de estas definiciones, defendemos la idea de que la escuela se convierte en un espacio propicio para la transformación del entorno, la participación de la comunidad educativa desde la base de unos principios democráticos. Así, se entiende que la eficacia del aprendizaje se consigue a través de la reconversión de los poderes profesionales $\mathrm{o}$ estamentales de la escuela (Elboj et al., 2002). Desde este enfoque se apuesta por el cambio, porque se considera que la organización del sistema educativo actual tiende a mantener las desigualdades y además no prepara al alumnado para los retos de la sociedad actual. Por eso, el centro educativo que se inicia como Comunidad de Aprendizaje sufre una transformación social, ya que se parte desde el cambio de todos los hábitos de comportamiento de familiares, profesorado, alumnado y comunidad. Se produce así un cambio de mentalidad para optar por el protagonismo de toda la comunidad en la educación.

Al contrario de lo que ocurría en las escuelas tradicionales donde se optaba por la adaptación y segregación del alumnado con necesidades distintas, el enfoque de las Comunidades de Aprendizaje apuesta por transformar el contexto. Además, en lugar de 
invertir más recursos, utiliza los existentes de forma inclusiva y los aumenta a través de la participación de miembros de la comunidad en las aulas (Díez-Palomar y Flecha, 2010). Se trata de una propuesta que se separa de los principios de la educación compensatoria, ya que no se fundamenta en el supuesto "déficit" a compensar del alumnado con desventajas sociales, sino en el incremento de la calidad de la enseñanza que reciben (Elboj et al., 2002) a través de la participación de toda la comunidad educativa.

Al mismo tiempo, las teorías internacionales se adhieren a las Comunidades de Aprendizaje, destacando las interacciones y la comunidad como componentes más importantes en el aprendizaje (Aubert, et al., 2008). Pues en palabras del mismo Vygotsky $(1979,89)$, "el aprendizaje activa una serie de procesos internos de desarrollo que son capaces de operar sólo cuando el niño está interactuando con personas de su entorno y en cooperación con sus compañeros". Es por ello que este modelo educativo trata de implicar a todas las personas que directa o indirectamente puedan influir en el desarrollo del aprendizaje del alumnado, desde el propio profesorado, hasta las familias, vecinos/as, organizaciones barriales o voluntariado.

De esta forma, se parte de la idea de que comunidad y escuela no son dos entes separados, ya que la escuela forma parte de la comunidad y se debe a ella. En esta línea, se hace posible instituir alianzas a través de los puntos comunes - antes de mayor desencuentro y ruptura- entre familia y escuela, alumnado y profesorado, alumnado y familias, y entre todos ellos y el conjunto de la sociedad local (Torres, 2004).

\subsection{Implantación de la Comunidad de Aprendizaje en un centro educativo.}

Para que un centro se transforme en Comunidad de Aprendizaje se pueden encontrar diferentes motivaciones. De esta forma, Rodríguez De Guzmán (2012) resalta que puede surgir a partir de la formación recibida en la Universidad, de la asistencia a un curso de formación permanente del profesorado, o también, a través de la urgencia por responder a una problemática concreta del centro educativo.

Por lo que a la hora de desarrollar el proceso que debe vivir un centro educativo para convertirse en Comunidad de Aprendizaje, partimos del estudio que han realizado diversas autorías desde el Centro Especial de Investigación en Teorías y Prácticas Superadoras de Desigualdad (en adelante CREA), a partir del cual se han sistematizado las fases que se deben llevar a cabo para que se produzca la transformación (Folgueiras, 2011). Se trata de ocho fases que se producirían durante un curso escolar y que se reflejan en el cuadro $\mathrm{n}^{\mathbf{o}} 1$. Así, vemos que Elboj et al., (2002) distinguen entre dos grandes fases: la puesta en marcha de la Comunidad de Aprendizaje, que puede durar un curso académico, y la consolidación, que se prolongará los años siguientes.

De esta forma, haciendo referencia al cuadro $\mathrm{n}^{\mathrm{o}} 1$, destacamos los procesos que debe seguir la Comunidad de Aprendizaje una vez finalizada la fase de puesta en marcha, ya que la fase de consolidación es permanente y simultánea a los cambios que se van produciendo, por lo que es necesario que se asegure la continuidad de los/as responsables que iniciaron la transformación. Además, resaltamos los procesos de investigación educativa que deben regir toda actuación que se quiera poner en marcha; los procesos de formación del profesorado y voluntarios que deben ser permanentes, siendo capaz de responder a las demandas de toda la comunidad educativa; y por último, la evaluación, implicando a toda la comunidad y recabando información a través de instrumentos adecuados, para poder tomar decisiones que sigan mejorando la escuela en la que nos encontramos (Elboj et al., 2002,cit. en Rodríguez De Guzmán, 2012). 
Cuadro 1. Puesta en marcha de una Comunidad de Aprendizaje por fases.

\section{Fase 1. SENSIBILIZACIÓN (Aproximadamente un mes)}

Sesiones iniciales de trabajo con los diferentes agentes (equipo directivo, profesorado, familiares, miembros de entidades, estudiantes, etc.) de la comunidad escolar.

$>\quad$ Estudio de la documentación entregada sobre las características del proyecto de comunidades con posterioridad a las jornadas informativas. propia comunidad.

Comunicado a la comunidad educativa sobre las conclusiones a las que ha llegado la

Fase 2. TOMA DE DECISIONES (Aproximadamente un mes)

\begin{tabular}{ll}
\hline & Decidir el inicio del proyecto. \\
\hline & Fase 3. SUEÑO (Entre uno v tres meses) \\
\hline & Reunirse en grupo para idear el centro educativo que se desea. \\
& $>\quad$ Llegar a un acuerdo sobre el modelo de centro que se pretende alcanzar. \\
& Contextualizar los principios de la comunidad de aprendizaje.
\end{tabular}

Fase 4. SELECCIÓN DE PRIORIDADES (Entre uno y tres meses)

\begin{tabular}{|l}
$\qquad \begin{array}{l}\text { Análisis de los datos obtenidos. } \\
\text { Selección de prioridades. }\end{array}$ \\
Fase 5. PLANIFICACIÓN (Entre uno v dos meses) \\
Crear comisiones de trabajo para llevar a cabo el plan de acción de cada prioridad.
\end{tabular}

Procesos para la continuidad de los procesos de transformación (Dos cursos escolares)

\begin{tabular}{|c|c|}
\hline & Fase 6. PROCESOS DE INVESTIGACIÓN \\
\hline$>$ & $\begin{array}{l}\text { Innovar para mejorar. Reflexión sobre la acción. } \\
\text { Experimentación del cambio. } \\
\text { Puesta en común de las experiencias llevadas a cabo y resultados. }\end{array}$ \\
\hline$>$ & Fase 7. PROCESOS DE FORMACIÓN \\
\hline$>$ & Formación solicitada por las comisiones de trabajo en función de los requerimientos \\
\hline & Formación de toda la comunidad de aprendizaje en núcleos de interés concreto. \\
\hline & Fase 8. PROCESOS DE EVALUACIÓN \\
\hline
\end{tabular}

Evaluación continua de todo el proceso.

Fuente: Elaboración propia a partir de Elboj et al., 2002. cit. en Folgueiras, 2011, p.264-265. 
La implantación de este modelo socioeducativo en cualquier centro escolar requiere la colaboración exhaustiva de todos los miembros de la comunidad educativa, y sin el trabajo conjunto y coordinado entre profesionales, especialmente de la mano de los/as maestros/as, este proyecto no sería posible. En este sentido, destacamos la necesidad de seguir formando a los/as futuros/as docentes hacia esta perspectiva socioeducativa de intervención escolar, pues en palabras de Orozco et al. $(2015,62)$

[...] somos conscientes de que procesos como éstos requieren esfuerzos y tiempos adicionales, pero ello no nos va a desanimar, y menos impedir soñar, pues los suelos son los que nos pueden permitir construir un futuro mejor, formando unos maestros realmente comprometidos con los tiempos que vivimos, conocedores de la realidad y creativos frente a los retos que la misma dinámica local, regional y mundial vaya exigiendo de todos nosotros.

Por otro lado, siguiendo el estudio de Alonso y Navarrete (2011), hay diversos aspectos que debemos tener en cuenta en la gestación de una Comunidad de Aprendizaje.

En primer lugar, el contexto, ya que éste es esencial para potenciar situaciones estimulantes y significativas de aprendizaje a través de la negociación, así como para redistribuir la participación del profesorado y el alumnado en todo el proceso de enseñanzaaprendizaje, desde su planificación hasta su evaluación.

Asimismo, la gestación de la comunidad debe partir de las necesidades sentidas por el alumnado, pues de esta forma es posible atender a las diferencias individuales y colectivas, identificando las soluciones y estableciendo metas. Pero además, este espacio fomenta la participación real de toda la comunidad educativa, por lo que cumple con su función transformadora.

Como consiguiente, a través de esta apertura a la participación, el aprendizaje se alcanza de forma democrática, lo que incentiva valores como la responsabilidad, la toma de decisiones, el respeto a los demás o la solidaridad, además de facilitar la adquisición de habilidades y actitudes para el trabajo grupal. Igualmente, en el momento en el que se gesta la Comunidad de Aprendizaje, aparecen diferentes espacios de reflexión, donde la comunidad educativa debe acordar roles, funciones, organización de los grupos, normas, líderes, etc. E incluso se potencia la reflexión de las propias conductas y de la forma misma de obtener el aprendizaje. Por lo que todo esto, constituye un proceso de reflexión y negociación constante, que como destacábamos, pone en juego muchas habilidades y valores, que hasta el momento no se visibilizan en la escuela.

Otro de los aspectos a tener en cuenta en la etapa de gestación de una Comunidad de Aprendizaje es que esto supondrá una refundación del centro con medidas administrativas especiales que deberán ser asumidas por la Consejería, y también una reorganización del funcionamiento del centro que a partir de este momento abre sus puertas al barrio. Por lo que es imprescindible que el equipo directivo esté plenamente sensibilizado y convencido de implementar la Comunidad de Aprendizaje en el centro, pues su liderazgo será esencial en la sensibilización y promoción de la participación de toda la comunidad educativa (Rodríguez De Guzmán, 2012).

\section{Sostenibilidad de una comunidad de aprendizaje desde la educación social.}

Como hemos visto, la Comunidad de Aprendizaje es un proyecto de éxito educativo que está obteniendo grandes resultados en diferentes comunidades autónomas de nuestro país e incluso se está extendiendo a otros países en Sudamérica y Centroamérica, como demuestra 
su presencia en Brasil o Chile (Ferreyra, 2011). No obstante, cuando hablamos del mantenimiento de este proyecto a lo largo del tiempo, es posible que encontremos algunos riesgos y dificultades, ya que como se expone en el artículo de Fórum IDEA (2002, 120), los proyectos de innovación y transformación social tienen, en algunas ocasiones, un efecto "bola de nieve", en el sentido de que al mismo tiempo que ilusionan y enriquecen una realidad educativa, pueden tener el efecto contrario, ya que un exceso de confianza en los buenos resultados del proyecto y la idea de que éste funcionará por sí mismo como inercia del éxito conseguido, pueden llevarle al fracaso.

Para contrarrestar este riesgo, destacamos el concepto de sostenibilidad, entendido como "la apuesta necesaria para que todo proyecto de innovación responda a la necesidad de ser intrageneracional (estar formado por distintos estamentos generacionales; entidades, administraciones, edades, género, clases sociales) así como intergeneracional (que pueda alcanzar, con los cambios necesarios a la siguiente generación)" (Fórum IDEA, 2002, 120). Por lo que, a continuación, presentamos algunas propuestas para la continuación de la Comunidad de Aprendizaje destacadas en el Fórum IDEA (Ibídem):

- Dejar la gestión del mayor número de actividades a cargo de las familias, ya que de esta forma podrán sentir el proyecto como una responsabilidad común, que les facilitará el intercambio de servicios y experiencias.

- Flexibilidad de la Comunidad de Aprendizaje, fomentando la idea de que este proyecto necesita de permanente evolución, adaptándose a los cambios que se vayan produciendo en la comunidad con el paso del tiempo.

- Asegurar la implicación del mayor número del claustro en todas las actividades y comisiones, sin crear especialistas que puedan convertirse en imprescindibles y hacer peligrar la continuidad del proyecto.

- Buscar nuevos proyectos y retos continuamente, de forma que se sigan generando acciones conjuntas entre todos los agentes de la comunidad.

- Potenciar la máxima implicación de toda la comunidad desde las primeras fases del proyecto, para que se sienta como un proyecto común, y no algo que desde la escuela se impone y no cuenta con el interés de todos.

- Darle continuidad al proyecto de la escuela en el instituto a través de la formación del ex alumnado como voluntariado del mismo.

- Evidenciar todo el trabajo a través de distintos medios, para que esto facilite la producción de un discurso transformador y ayude a difundir y compartir la experiencia a otros espacios.

- Conocer otras experiencias innovadoras y buscar la colaboración y el trabajo en red con otros centros que puedan enriquecer la realidad escolar.

- Asegurar la formación del voluntariado para que su acción cobre sentido y pueda ser revisada y evaluada cada curso.

Por otro lado, además, entre estas acciones de sostenibilidad, no podemos olvidar el papel tan importante que puede jugar la universidad en la Comunidad de Aprendizaje de un centro escolar. Así, podemos citar la experiencia de CREA en la Universidad de Barcelona, que desde el inicio ha estado investigando cómo desarrollar una dinámica que fomenta el éxito educativo para todo el alumnado, y elabora, así, el modelo de Comunidad de Aprendizaje en coordinación con todos los centros educativos que quisieran llevarlo a cabo (Rodríguez De Guzmán, 2012). Dicha experiencia se postula como una acción de sostenibilidad, ya que el CREA a través de sus investigaciones ha desarrollado una reflexión y evaluación de los distintos proyectos realizados en cada contexto educativo, que les ha facilitado la mejora continua y el establecimiento de nuevas metas por parte de los centros educativos. Asimismo, ha estado presente en todas las fases que se han llevado a cabo en los 
centros educativos que se han transformado en Comunidad de Aprendizaje, favoreciendo la formación permanente y colaborando directamente en sus acciones. Esto muestra como la universidad puede desarrollar proyectos que tengan una incidencia social y que potencien la transformación del entorno.

En la misma línea citar a la Universidad de Sevilla, que colabora con las Comunidades de Aprendizaje de la provincia de Sevilla, a través de la formación por parte del profesorado universitario y personal investigador durante la fase de sensibilización, y también facilita el voluntariado y prácticas de estudiantes universitarios en las distintas Comunidades de Aprendizaje (Díez-Palomar y Flecha, 2010).

Sin embargo, si nos planteamos qué lugar ocupan las actuaciones educativas de éxito que las Comunidades de Aprendizaje estudian, en los planes universitarios de los profesionales de la educación o de la misma formación del profesorado, podemos evidenciar que aunque existen bloques donde podemos incluir entre sus contenidos estas prácticas, en la realidad no aparecen. Además, se observa la ausencia de estudios más actualizados que presenten experiencias educativas de éxito, respaldadas por la comunidad científica y referenciadas en publicaciones especializadas (Rodríguez De Guzmán, 2012).

Esta situación se presenta como una debilidad, ya que si hemos citado la importante labor de impulso y sostenibilidad que puede tener la universidad en los centros educativos a través del acercamiento a la experiencia de CREA, por otro lado, podemos observar la falta de coherencia real de los planes de estudio de los profesionales de la educación en muchas universidades con las experiencias educativas de éxito. Esto desemboca en una ausencia de formación de los maestros, que no tienen la oportunidad de estudiar prácticas educativas que ya se están llevando a cabo en las escuelas, y que se ajustan a las demandas que plantea la sociedad cambiante en la que vivimos (Ibídem - Rodríguez De Guzmán, 2012).

Asimismo, esta debilidad de los planes de estudio universitarios tiene una repercusión en las motivaciones y barreras que el equipo docente manifiesta cuando se aborda un nuevo proyecto educativo en su centro. Por lo que podemos encontrar factores que influyen en la adopción de un proyecto innovador, como pueden ser la congruencia entre un proyecto y la práctica actual o la cercanía entre las concepciones del profesorado y la filosofía de la propuesta. Del mismo modo, el valor que se le da a los cambios que hay que introducir o el dominio operativo de un proyecto (Fullan, 2004). También podemos nombrar la percepción de acompañamiento y el asesoramiento colaborativo como factores a tener en cuenta entre el profesorado (Domingo, 2005), así como las expectativas de obtener buenos resultados con el desarrollo del proyecto (Abrami et al., 2004).

En la misma línea, destacar como otra fortaleza o debilidad los sentimientos que aparecen cuando una institución educativa se embarca en un proyecto innovador como son las Comunidades de Aprendizaje, es decir, nos referimos al rol que ocupa las emociones como el entusiasmo, el miedo a perder el control del alumnado, la sensación de correr riesgos, que las acciones termine en fracaso o el rechazo a implicarse más de lo necesario (Day, 2006).

Todos estos factores tienen una relación directa con la sensibilización y formación del profesorado, por lo que deben tenerse en cuenta cuando se inicia la transformación de un centro a Comunidad de Aprendizaje. Pero también es importante tenerlos en cuenta en la sostenibilidad del proyecto, ya que la falta de formación, la inestabilidad de la plantilla docente en los centros públicos, el desajuste de las expectativas, la sobrecarga de trabajo o sobre-implicación ante la falta de disposición de otros, el sentimiento de soledad y falta de apoyo, etc., pueden hacer peligrar la continuidad de la Comunidad de Aprendizaje en la institución educativa. 


\subsection{La Educación Social en la sostenibilidad de la Comunidad de Aprendizaje}

En primer lugar, cabe destacar la idea de tomar la educación social como perfil profesional de referencia en las Comunidades de Aprendizaje con la finalidad de que este modelo socioeducativo se desarrolle y se mantenga en tiempo y forma dentro de cualquier institución educativa. En este sentido, especificamos que entendemos por Educación Social aquel

[...] Derecho de la ciudadanía que se concreta en el reconocimiento de una profesión de carácter pedagógico, generadora de contextos educativos y acciones mediadoras y formativas, que son ámbito de competencia profesional del educador social, posibilitando:

- La incorporación del sujeto de la educación a la diversidad de las redes sociales, entendida como el desarrollo de la sociabilidad y la circulación social.

- La promoción cultural y social, entendida como apertura a nuevas posibilidades de la adquisición de bienes culturales, que amplíen las perspectivas educativas, laborales, de ocio y participación social" (ASEDES, 2007, 12; López Zaguirre, 2013, 1)

En cuanto a la concreción de la Educación Social dentro de este proyecto educativo de éxito como son las Comunidades de Aprendizaje, continuamos defendiendo su presencia en la educación formal, ya que en la práctica y realidad de nuestro país seguimos observando una gran ausencia, y es que la presencia de los/as educadores/as sociales en los centros educativos es mínima, restringiéndose a zonas catalogadas como conflictivas, marginales o simplemente zonas de transformación social. Es por ello que, siguiendo a Valls (2005), defendemos que el proyecto de Comunidades de Aprendizaje cambia radicalmente con el rol del/la educador/a social en el centro, ya que a partir de la creación de comisiones mixtas, dicho profesional tiene la oportunidad de acercarse a las familias, al contexto, al barrio... y responder a las nuevas necesidades que vayan surgiendo en la comunidad educativa, especialmente, en todo el alumnado.

En la actualidad el Consejo General de Colegios de Educadores y Educadoras Sociales (CGCEESS), creado por la Ley 41/2006 de 26 de diciembre de Creación del Consejo General de Colegios Oficiales de Educadoras y Educadores Sociales (BOE 309 de 27 diciembre 2006), sigue reivindicando que dicho profesional debe estar dentro del equipo docente, específicamente, debe formar parte del "Claustro del Profesorado y/o ser un miembro del Departamento de Orientación o de Atención a las necesidades educativas especiales, bajo la dependencia del Jefe de Departamento y en colaboración con el equipo directivo y tutores y asumir la función de enlace y mediación directa con todos los recursos sociales de la comunidad" (CGCEESS, 2015, 19 citado en Terrón, Cárdenas y Rodríguez, 2016).

Así, siguiendo a Castillo (2013), debemos apostar por la Educación Social dentro de la institución escolar como una pieza fundamental en el engranaje educativo, que no se sitúa como una figura de emergencia para resolver problemas emergentes, sino que se postula como agente de cambio, con las capacidades necesarias para conseguir una interconexión con las familias, actuando como mediador entre el centro, las familias y el entorno social, como animador sociocultural, como dinamizador de grupos, como detector de factores de riesgo, etc.

La participación de la comunidad en todos los niveles de decisión, planificación y actividad del centro educativo, favorece la aparición de nuevos recursos, asumiéndose una nueva función de coordinación y dinamización por parte de la propia comunidad, que antes 
hacía el educador/a social en solitario. Lo mismo ocurre con la mediación y resolución de conflictos, al aparecer una comisión de convivencia que apoya la tarea del profesional y la extiende al barrio. Esto favorece la generación de nuevas colaboraciones de otras entidades y asociaciones con la escuela (Valls, 2005).

Con respecto a las funciones que el/la educador/a social, según la misma autoría, puede desarrollar en un centro educativo, vemos como la Comunidad de Aprendizaje le ofrece más oportunidades mediante la nueva organización interna y el diferente modo de establecer la relación entre la educación dentro y fuera de los centros escolares, lo que convierte al profesional de la Educación Social en un elemento imprescindible para afrontar situaciones de vulnerabilidad y riesgo socioeducativo, donde el equipo docente no posee las competencias y habilidades necesarias para una intervención social. Por otra parte, como otra línea más de intervención socioeducativa, se le abre un nuevo camino hacia el desarrollo comunitario y la promoción de la educación a lo largo de la vida para con la comunidad educativa.

En línea con el perfil profesional del/a educador/a social, como posibles funciones a desarrollar en las Comunidades de Aprendizaje, resaltamos aquellas referentes al análisis de la realidad, análisis de las situaciones-problemas, la detección y priorización de necesidades (manifiestas y latentes), así como sus causas y consecuencias, las relaciones y comunicaciones, la gestión, organización y participación en la vida comunitaria y cotidiana, la promoción de actividades socioeducativas, el diseño, ejecución y evaluación de proyectos socioeducativos (Valls, 2005). Por su parte, Merino (2013) añade que el/la educador/a social desde su ámbito profesional debe intervenir para fortalecer la participación de toda la comunidad educativa en si realidad, no sólo escolar, sino también social y desde una perspectiva preventiva.

Relacionado con las funciones destacamos que dentro del ámbito escolar, y por consiguiente como tareas en el marco de las Comunidades de Aprendizaje, según la Junta de Andalucía (Instrucciones de 17 de septiembre de 2010) los diversos campos de actuación del/la educador/a social que se recogen son los referentes a la convivencia y resolución de conflictos; a la prevención, el seguimiento y control del absentismo escolar; a la dinamización y participación familiar y comunitaria; al acompañamiento y tutorización en situaciones de riesgo y/o vulnerabilidad para el alumnado; a la educación en valores y competencia social y, por último, a la intervención educativa con minorías étnicas y educación intercultural.

Sólo de esta forma y en el contexto educativo, la labor del profesional de la Educación Social continuará teniendo mayor repercusión social, y a seguir siendo más respaldada y reconocida entre su comunidad. Constituyendo así, un nuevo impulso para transformar la realidad hacia la inclusión social, además de una oportunidad para incidir en la mejora de una sociedad donde todos los niños y niñas puedan desarrollarse plenamente, tanto personal como académicamente.

\section{Conclusiones}

A través de estas líneas, hemos intentado presentar unas propuesta de desarrollo y sostenibilidad desde la educación social para las Comunidades de Aprendizaje en instituciones educativas, lo que resulta bastante innovador, ya que si revisamos la bibliografía, observamos como existe una ausencia de documentación y estudios relacionados con la sostenibilidad de estos proyectos, a pesar del hecho de que en muchos centros han ido desapareciendo con el paso de los años, y en los que además se resalta una falta de conocimientos por parte de la misma comunidad para hacer frente a esta situación. 
Esta idea puede derivar en la conclusión de que la transformación educativa es posible pero difícil e incómoda para diferentes sectores de la población. Es como un sueño, una utopía de la educación y, por supuesto, del sistema educativo actual. Es un camino que se empieza a visibilizar a nivel internacional, pero que debemos continuar con un arduo trabajo de sensibilización, asesoramiento científico, formación, experiencias docentes... y, en gran medida, intereses, ilusiones y emociones comunes para creer en la transformación de los centros escolares en pro de la inclusión educativa y la igualdad de oportunidades (Morlà, 2015).

También hemos reflexionado sobre la figura del profesional de la Educación Social como pieza clave que puede y debe impulsar estos procesos socioeducativos que atañen a toda la comunidad educativa, ya que si analizamos los centros escolares de nuestra comunidad autónoma que cuentan con esta figura profesional, encontraremos que es más una excepción que una regla. Por lo que desde esta publicación se resalta la especial perspectiva que el/a educador/a social incorpora a los centros educativos, presentándose como una herramienta necesaria para construir una escuela más justa e igualitaria, una escuela transformadora, donde se promuevan los valores de la solidaridad, la diversidad y el respeto.

En este sentido, es imprescindible destacar que esta propuesta fomenta la valorización de la profesión de la educación social dentro del campo educativo, y es una forma de sensibilizar a la comunidad educativa sobre la importante labor que pueden realizar estos profesionales en los centros escolares. Esto resulta totalmente significante para la Educadora Social, que ve abierto un campo de actuación con la comunidad que le permitirá fomentar su empoderamiento frente a las necesidades y problemáticas que se desprenden del propio entorno. Asimismo, recalcamos que la incorporación del/a educador/a social al ámbito formal debe de ser impulsada por parte del Estado, desarrollando normativas socioeducativas a nivel local que garanticen y permitan ser una realidad en los centros escolares de nuestro país.

Como conclusión final, siguiendo a investigaciones recientes (Orozco et al., 2015; Redondo, 2015; Morlá, 2015) las Comunidades de Aprendizaje muestran resultados positivos tanto a nivel académico como en la convivencia escolar. Hablamos de diferentes profesionales y personas que se involucran para que una escuela se transforme en Comunidad de Aprendizaje. En este arduo camino son muchas las personas que sueñan por alcanzar el mismo objetivo en su centro escolar: más cohesión, más inclusión, más diálogo y comunicación, más valores hacia el respeto y la solidaridad, más democracia participativa, más desarrollo comunitario, más reflexión y visión crítica, más conocimiento mutuo... en definitiva, más transformación que garantice el éxito académico de todos y todas los que participamos en la Comunidad de Aprendizaje.

\section{Referencias Bibliográficas}

Abrami, P., Poulsen, C. y Chambers, B. (2004). Teacher Motivation to Implement an Educational Innovation: Factors differentiating users and non-users of cooperative learning. Educational Psychology, 24(2), 201-216.

Aubert, A., Flecha, A., García, C., Flecha, R. y Racionero, S. (2008). Aprendizaje dialógico en la sociedad de la información. Barcelona: Hipatia Editorial.

Consejería De Educación, Junta De Andalucía (2010). Instrucciones de la Dirección General de Participación e Innovación Educativa por las que se regula la intervención del educador y educadora social en el ámbito educativo. Recuperado de: 
http://www.juntadeandalucia.es/educacion/webportal/abacoportlet/content/bcf29fb5-6823-4fe2-a0f4-0277cbeb2365.

Díez-Palomar, J. Y Flecha, R. (2010). Comunidades de aprendizaje: Un proyecto de transformación social y educativa. Revista Interuniversitaria de de Formación para el Profesorado, 67 (24,1), 19-30.

Domingo, J. (2005). El asesoramiento al centro educativo: Colaboración y cambio en la institución. Barcelona: Ediciones Octaedro.

Elboj, C., Puigdellívol, I., Soler, M. y Valls, R. (2002) Comunidades de aprendizaje: Transformar la educación. Barcelona: Graó.

Ferrer, G. (2005). Hacia la excelencia educativa en las comunidades de aprendizaje: participación, interactividad y aprendizaje. Educar, 35, 61-70.

Ferreyra, A. (2011). Motivaciones y barreras a la transformación de un centro educativo a comunidad de aprendizaje. Tendencias Pedagógicas, 17, 34-50.

Folgueiras, P. (2011). Las Comunidades de Aprendizaje: La escuela de adultos de la Verneda: Una experiencia de comunidades de aprendizaje. Tendencias Pedagógicas, 18, 252267.

Fórum Idea (2002). Comunidades de aprendizaje: participación, calidad y transformación social. Educar, 29, 103-121.

Fullan, M. (2004). Las fuerzas del cambio: La continuación. Madrid: Editorial Akal.

Gómez, A., Mello, R., Santa Cruz, I. y Sordé, T. (2010) De las experiencias de Comunidades de Aprendizaje a las políticas basadas en sus éxitos. Revista Interuniversitaria de Formación del Profesorado, 67, 24(1), 113- 126.

Mórlà, T. (2015). Comunidades de Aprendizaje, un Sueño que hace más de 35 años que Transforma Realidades. Social and Education History, 4(2), 137-162. doi: 10.17583/hse.2015.1496

Rodríguez De Guzmán, J. (2012). Comunidad de aprendizaje y formación del profesorado. Tendencias Pedagógicas, 19, 68-86.

Terrón Caro, T., Cárdenas Rodríguez, R., y Rodríguez Casado, Ma R. (2016) Educación Intercultural Inclusiva. Funciones de los/as Educadores/as en instituciones educativas. Pedagogía Social. Revista Interuniversitaria (Artículo aceptado, pendiente de publicación).

Torres, R. (2004) Comunidad de aprendizaje: Repensando lo educativo desde el desarrollo local y desde el aprendizaje. Documento presentado en el "Simposio Internacional sobre Comunidades de Aprendizaje". Barcelona Fórum 2004. Barcelona 2001. Recuperado de: http://www.inafocam.edu.do/cms2/data/formacion/comunidades_y_aprendizaje.pdf

Valls, R. (2000). Comunidades de Aprendizaje: Una práctica educativa de aprendizaje dialógico para la sociedad de la información. (Tesis doctoral. Programa de 
Doctorado: "Pedagogía Social y Políticas Sociales"). Departamento de Teoría e Historia de la Educación. Universidad de Barcelona.

Valls, R. (2005). Los educadores y educadoras sociales en las comunidades de aprendizaje. Revista de Educación Social. Infancia y Educación Social, 4. Recuperado de: http://www.eduso.net/res/?b=7\&c=53\&n=145

Vygotsky, L. (1979). El desarrollo de los procesos psicológicos superiores. Barcelona: Crítica. 
